\title{
Podoplanin expression in odontomas: clinicopathological study and immunohistochemical analysis of 86 cases
}

\author{
Patricia González-Alva1), Harumi Inoue ${ }^{1)}$, Yuji Miyazaki ${ }^{1)}$, Hozumi Tsuchiya'), \\ Yoshihiro Noguchi ${ }^{1)}$, Kentaro Kikuchi ${ }^{1)}$, Fumio Ide ${ }^{1)}$, Sachiyo Ishihara ${ }^{2)}$, \\ Tadashi Katayama ${ }^{2}$, Hideaki Sakashita ${ }^{3)}$ and Kaoru Kusama ${ }^{1)}$ \\ ${ }^{1)}$ Division of Pathology, Department of Diagnostic and Therapeutic Sciences, \\ Meikai University School of Dentistry, Saitama, Japan \\ ${ }^{2}$ Division of Operative Dentistry, Department of Restorative and Biomaterials Sciences, \\ Meikai University School of Dentistry, Saitama, Japan \\ ${ }^{3)}$ Division of Oral \& Maxillofacial Surgery II, Department of Diagnostic and Therapeutic Sciences, \\ Meikai University School of Dentistry, Saitama, Japan
}

(Received 10 November 2010 and accepted 27 January 2011)

\begin{abstract}
Podoplanin, a sialomucin-like transmembrane glycoprotein, is currently used as a specific marker for lymphatic vessels. However, podoplanin expression has also been linked to tooth development. To investigate the expression of podoplanin in odontomas, 86 tissue samples were classified and then analyzed using immunohistochemical methods. Formalin-fixed, paraffin-embedded specimens were collected and classified, followed by immunohistochemical examination. The majority of the odontomas $(66.3 \%)$ were the compound type, and the remainder $(33.7 \%)$ were the complex type. The patients ranged in age from 2 to 89 years (mean, 23.9 years), and $45(52.3 \%)$ of them were male and $41(47.7 \%)$ were female. The most common location for complex odontomas was the molar region of the mandibular bone, and that for compound odontomas was the maxillary incisor region. Immunohistochemistry revealed that developing and mature odontoblasts, Tomes' fibers, and pulp cells near podoplanin-positive odontoblasts were positive for
\end{abstract}

Correspondence to Dr. Patricia González-Alva, Division of Pathology, Department of Diagnostic and Therapeutic Sciences, Meikai University School of Dentistry, 1-1 Keyakidai, Sakado, Saitama 350-0283, Japan

Tel: +81-49-279-2773

Fax: +81-49-286-6101

E-mail: patriciagoa@yahoo.com.mx \& patricia@dent.meikai.ac.jp podoplanin. In addition, podoplanin positivity was evident in secretory ameloblasts, but not in mature ameloblasts. The pattern of podoplanin expression in odontomas corresponds to development of the tooth germ, and appears to be influenced by the stage of differentiation of the lesion, suggesting that the protein may participate in the process of differentiation. ( $J$ Oral Sci 53, 67-75, 2011)

Keywords: podoplanin; odontoma; complex odontoma; compound odontoma; odontogenic tumor.

\section{Introduction}

Odontomas are recognized as hamartomatous odontogenic lesions because they are composed of both epithelial and ectomesenchymal components, and even when the cells appear normal morphologically, their structural arrangement is apparently defective (1-3). The 2005 World Health Organization (2) classification of odontogenic tumors includes two different types of odontoma: complex and compound odontoma $(1,2)$. The morphology of odontomas provides an alternative model for studying the formation of dental tissues, because different stages of maturation are present simultaneously.

Human podoplanin is a type- 1 transmembrane sialomucin-like glycoprotein comprising 162 amino acids. 
Podoplanin was originally detected in puromycin-induced nephrosis on the surface of rat podocytes as a $38-\mathrm{kDa}$ mucoprotein $(4,5)$. As podoplanin is expressed on lymphatic, but not blood vessel endothelium, it is now used as a specific marker for lymphatic vessels (4). The physiological function of podoplanin is still unknown. Podoplanin-deficient mice die at birth due to respiratory failure and lymphedema $(4,6)$. These findings suggest a role of podoplanin in tissue development.

In addition, podoplanin expression has been reported in other normal tissues and pathological conditions, such as squamous cell carcinoma of the lung, uterine cervix, and head and neck, oral leukoplakia, pleomorphic adenoma, and odontogenic tumors (7-12). With respect to tooth development, immunohistochemical studies of mouse and human tooth germ have revealed that podoplanin expression varies according to developmental stage $(5,12)$, the expression being evident on the inner enamel epithelium and differentiating ectomesenchymal cells. Later in tooth development, podoplanin is expressed in odontoblasts, Tomes' fibers, and pulp cells associated with odontoblasts, but it is reduced or lost in ameloblasts after completion of crown formation $(5,12)$. The available data suggest a possible role of podoplanin in tooth development (5).

Furthermore, a previous study conducted in our laboratory has demonstrated positivity for podoplanin in basal cell extensions (13), which are frequently observed in gingival epithelium but not in other types of oral epithelia, and are thought to possess the characteristics of odontogenic epithelium (14).

In the present study using immunohistochemical methods, we analyzed podopanin expression in 86 cases of odontoma diagnosed at our department. The clinical and histopathological features of these cases were also taken into consideration, following the criteria of the WHO classification of odontogenic tumors. A characteristic distribution of podoplanin was found in these tumors, and the possible role of the protein in odontogenic tumors and tooth development was discussed.

\section{Materials and Methods}

\section{Tissue samples}

Cases of odontoma diagnosed at the Division of Pathology, Department of Diagnostic and Therapeutic Sciences, Meikai University School of Dentistry, between 1982 and 2004 were used in this study. Tissue sections stained with hematoxylin-eosin (H \& E) were examined, and histological diagnosis was based on the criteria of the WHO classification. In total, 86 cases were available for study.

Data on patient age, gender and lesion site were obtained from the information submitted with the biopsy request forms. The study protocol was reviewed and approved by the Research Ethics Committee of Meikai University School of Dentistry (A0832).

\section{Immunohistochemistry}

Serial sections of odontoma specimens were deparaffinized, and immersed in methanol with $0.3 \%$ (v/v) hydrogen peroxide for $15 \mathrm{~min}$ at room temperature to block endogenous peroxidase activity. After washing with running water and phosphate-buffered saline (PBS; $0.01 \mathrm{M}$ phosphate buffer, $0.15 \mathrm{M} \mathrm{NaCl}, \mathrm{pH} 7.4)$, the sections were heated in a microwave oven at high power while immersed in $0.01 \mathrm{M}$ citrate buffer ( $\mathrm{pH}$ 6.0) for $5 \mathrm{~min}$, and then for $15 \mathrm{~min}$ at low power for antigen retrieval. All sections were then incubated in $2 \%(\mathrm{w} / \mathrm{v})$ bovine serum albumin (BSA) for $20 \mathrm{~min}$ to block non-specific reactions. Appropriately diluted antibody against podoplanin (D2-40; Dako North America, Inc., Carpinteria, CA, USA; dilution 1:50) was applied to each section for $1 \mathrm{~h}$ at room temperature. The sections were then incubated with MAX-PO (Nichirei Bio, Inc., Tokyo, Japan) for $30 \mathrm{~min}$, and the reaction products were visualized by immersing the sections in freshly prepared $0.05 \%$ diaminobenzidine solution (75 mg diaminobenzidine tetrahydrochloride, $50 \mu \mathrm{l} 30 \% \mathrm{H}_{2} \mathrm{O}_{2}$, $150 \mathrm{ml} \mathrm{0.05} \mathrm{M} \mathrm{Tris-HCl} \mathrm{buffer} \mathrm{pH}$ 7.6) for 6-8 min. Nuclei were lightly stained with Mayer's hematoxylin. Lymphatic vessels and osteocytes were used as internal positive controls. To compare the expression of podoplanin with mature dental tissues, 6 impacted teeth were included in the present study.

\section{Results}

\section{Clinicopathological findings}

A total of 86 patients diagnosed as having odontoma was selected. They ranged in age from 2 to 89 years (mean 23.9 years) at the time of diagnosis, with a peak incidence in the second decade of life (Table 1). There were 45 males $(52.3 \%)$ and 41 females $(47.7 \%)$, with a male to female ratio of 1.09:1. Table 2 shows the site distribution of the odontomas. The most common location was the maxillary incisor region (40.7\%), followed by the mandibular incisor region $(18.6 \%)$.

Histologically, complex odontomas accounted for $33.7 \%$ (29 cases) of the total odontoma pool in the present study. The mean patient age at the time of diagnosis was 30.5 years (range, 8 to 71 years). Figure 1 shows the age group/gender distribution at the time of diagnosis for complex odontoma. Complex odontoma occurred in 11 male and 18 female patients, with a male to female ratio of 1:1.6 (Fig. 1). The majority (55.2\%, 16 cases) occurred 
Table 1 Age and gender distribution of the 86 odontoma cases

\begin{tabular}{cccc}
\hline Age & Male & Female & Total $(\%)$ \\
\hline $0-9$ & 8 & 7 & $15(17.4)$ \\
$10-19$ & 20 & 13 & $33(38.4)$ \\
$20-29$ & 9 & 6 & $15(17.4)$ \\
$30-39$ & 3 & 7 & $10(11.6)$ \\
$40-49$ & 1 & 1 & $2(2.32)$ \\
$50-59$ & 2 & 4 & $6(6.97)$ \\
$60-69$ & 1 & 1 & $2(2.32)$ \\
$70-79$ & 1 & 1 & $2(2.32)$ \\
$80-89$ & 0 & 1 & $1(1.7)$ \\
\hline Total & 45 & 41 & 86 \\
$(\%)$ & $(52.3)$ & $(47.7)$ & $(100)$ \\
\hline
\end{tabular}

Table 2 Distribution of the odontoma cases within the jaws

\begin{tabular}{l|c|c|c}
\hline \multicolumn{1}{c|}{ Location } & $\begin{array}{c}\text { Complex } \\
\text { odontoma }\end{array}$ & $\begin{array}{c}\text { Compound } \\
\text { odontomas }\end{array}$ & Total (\%) \\
\hline Mandibular incisor & 2 & 14 & $16(18.6)$ \\
Mandibular premolar & 0 & 8 & $8(9.3)$ \\
Mandibular molar & 12 & 3 & $15(17.4)$ \\
Maxillary incisor & $7^{*}$ & 28 & $35(40.7)$ \\
Mandibular premolar & 1 & $3^{* *}$ & $4(4.7)$ \\
Mandibular molar & 7 & 1 & $8(9.3)$ \\
\hline *, Including 2 cases of extended location from incisor to premolar; \\
**, including one case of extended location from the premolar to \\
the molar area.
\end{tabular}

before the age of 30, with a peak incidence in the second decade of life. The most common location for complex odontomas was the posterior region of the mandibular bone $(41.4 \%, 12$ cases), followed by the posterior region of the maxillary bone (27.6\%, 8 cases) (Table 2$)$.

Microscopically, the lesions consisted primarily of a mass of hard dental tissue with no apparent tooth-like morphology (Fig. 3A). However, the degree of morphodifferentiation varied from lesion to lesion, and some cases did include better-ordered, tooth-like structures. Ghost cells were found in $41.4 \%$ ( 12 cases) of the 29 complex odontomas, being present at different locations: next to tubular dentin, at sites where enamel would be expected; adjacent to remnants of, or surrounded by, enamel matrix, within calcified masses, in contact with bone; in contact with ameloblasts or adjacent to small rests of odontogenic epithelium. Many of the ghost cells were calcified or were embedded in an amyloid-like matrix (Figs. 3C, D).

Compound odontomas accounted for $66.3 \%$ (57 cases) of the 86 odontoma cases in the present series. The mean patient age at the time of diagnosis was 20.4 years (range, 7 to 89 years). Figure 2 shows the age group/gender

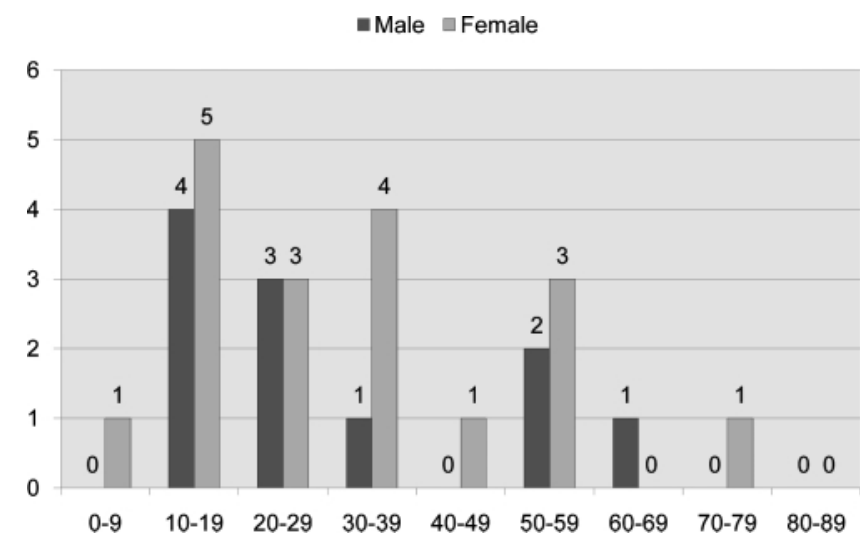

Fig. 1 Bar graph indicating patient ages at diagnosis of complex odontomas according to gender. There were 11 male (mean age 28.7 years) and 18 female (mean age 31.5 years) patients, the gender ratio being 1:1.6.

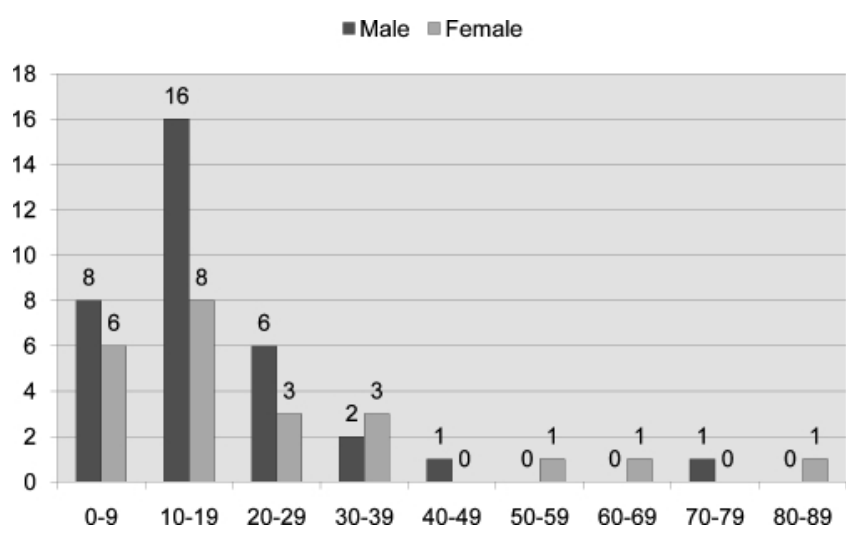

Fig. 2 Bar graph indicating patient ages at diagnosis of compound odontomas according to gender. There were 34 male (mean age 18.2 years) and 23 female (mean age 23.9 years) patients, the gender ratio being 1.5:1.

distribution at the time of diagnosis of complex odontoma in the present series. Compound odontoma occurred in 34 males and 23 females, with male to female ratio of 1.5:1. The majority of the cases $(66.7 \%, 38$ cases) occurred before the age of 20 years, with a peak in the second decade of life. The most common location for compound odontomas was the anterior maxillary bone $(49.1 \%, 28$ cases), followed by the anterior mandibular bone (24.6\%, 14 cases) (Table 2).

All the specimens of compound odontoma showed different degrees of histo- and morphodifferentiation, and in all of them a number of morphologically recognizable teeth were evident (Fig. 3B). In most of the cases, dentin formed the bulk of the specimen and was covered by an 

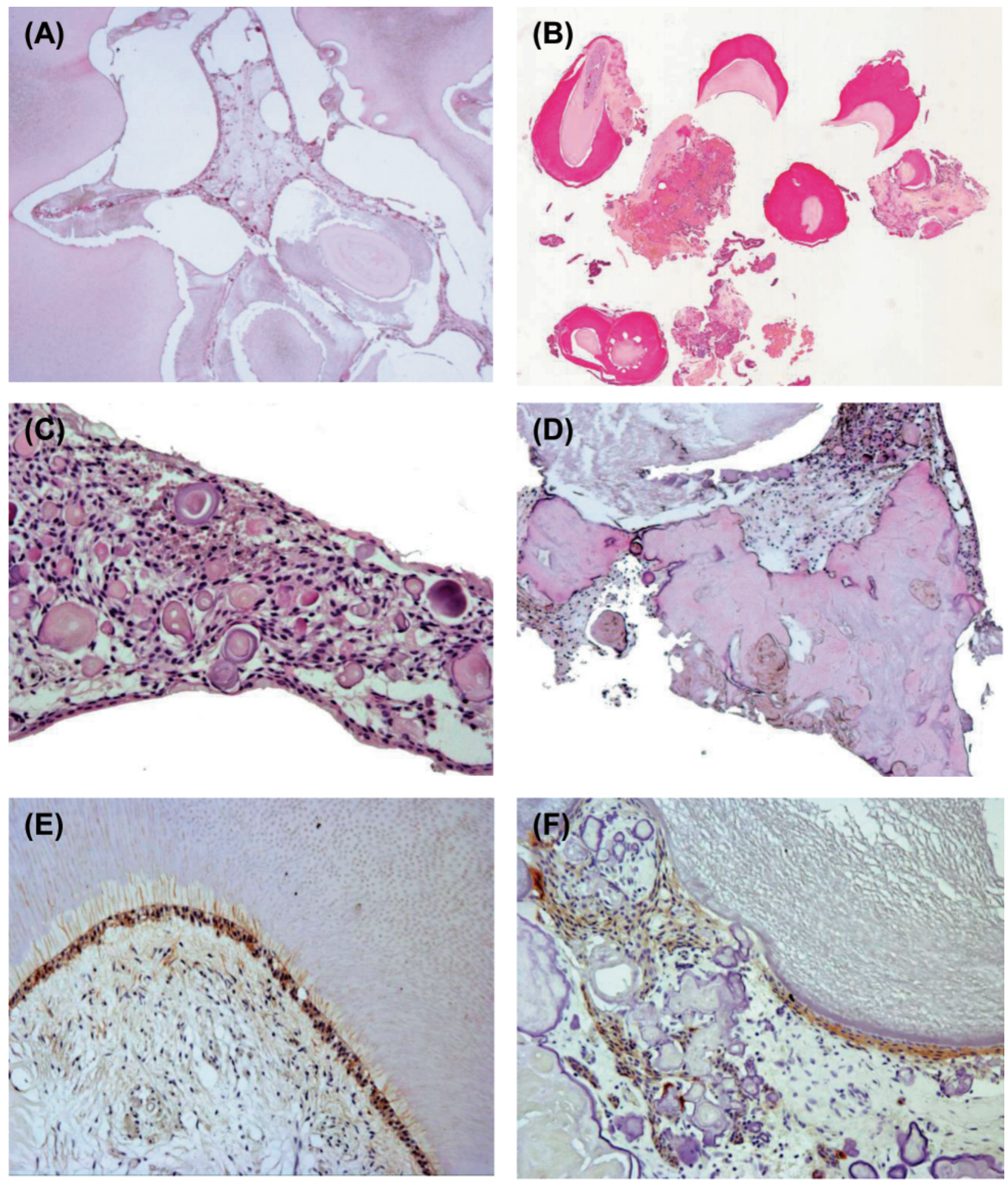

Fig. $3 \mathrm{H}$ \& E staining of complex (A, C) and compound (B, D) odontoma. (A) Odontogenic tissues are arranged in a disorganized pattern $(\times 2)$. (B) One of several malformed teeth, odontogenic epithelium and immature enamel can be recognized $(\times 2)$. $(\mathrm{C})$ Ghost cells at different stages of calcification, some showing circular calcification similar to Liesegang's rings $(\times 4)$; odontogenic epithelium is abundant. (D) Ghost cells are evident within a large area of dystrophic calcification $(\times 2)$. Immunohistochemical reactivity for podoplanin in compound $(\mathrm{E})$ and complex (F) odontoma. (E) Expression of podoplanin is evident in columnar odontoblasts, dentinal fibers, and some pulp cells adjacent to odontoblasts $(\times 10)$. (F) Odontogenic epithelium resembling the enamel organ is positive for podoplanin. In addition, immature enamel associated with ameloblast-like cells is also positive for podoplanin (×10). 



Fig. 4 Immunohistochemical reactivity for podoplanin in odontomas (A,B,C,D,E) and an impacted tooth (F). (A) Stellate reticulum-like cells in developing odontoma are negative or weakly positive $(\times 20)$. (B) Enamel epithelium of the cervical loop is strongly positive in areas of cell-cell contact $(\times 10)$. (C) Epithelial columnar cells with centrally located nuclei are strongly positive for podoplanin $(\times 20)$. (D) Ghost cells with granular cytoplasm are weakly positive for podoplanin, and the epithelial component associated with these cells is strongly positive $(\times 4)$. (E) Ghost cells entrapped within tubular dentin or calcified materials show a partially positive reaction for podoplanin $(\times 10)$. $(\mathrm{F})$ Impacted tooth showing positivity for podoplanin in superficial odontoblasts, tubular dentin, and pulp cells associated with odontoblasts $(\times 2)$. 
area of decalcified enamel. Dysplastic calcified tissues in less differentiated odontomas presented different degrees of mineralization and organization. Ghost cells were present at the enamel surface or in the ameloblastic epithelium in $66.7 \%$ (38 cases) of the compound odontomas, and these cells showed different degrees of nuclear pyknosis and granular or clear cytoplasm; calcifications in individual cells or clusters of cells, characterized by extremely fine basophilic granularity, were present in some cases.

In one of the cases of compound odontoma affecting a 7-year-old male, the lesion was localized in the overlying alveolar mucosa of the left mandibular incisor. Histopathological examination revealed a surface of stratified squamous epithelium covering a developing tooth germ composed of dental papilla, stellate reticulum, stratum intermedium, ameloblasts, dentin and ghost cells, the lesion was diagnosed as "developing odontoma".

\section{Immunohistochemical findings}

Podoplanin immunoreactivity was found in columnar odontoblasts adjacent to the dentin matrix, particularly in compound-type odontomas. The immunopositive odontoblasts were arranged in a palisade pattern adjacent to predentin or dentin (Fig. 3E). Tomes' fibers extending through the dentinal tubules from the odontoblasts also showed immunoreactivity, and pulp cells adjacent to the odontoblasts were positive for podoplanin in some cases (Fig. 3E). In addition, in complex odontomas, sparse flat cells adhering to dentin-like, irregular dentin tubules or Liesegang's ring calcifications were also positive for podoplanin (Fig. 3F). In developing odontomas, and in less differentiated odontomas, stellate reticulum-like cells were negative or weakly positive for podoplanin (Fig. 4A); in addition, when enamel epithelium of the cervical loop was present, strong podoplanin expression was detected in areas of cell-cell contact (Fig. 4B).

The presence of prismatic enamel was observed in both compound and complex odontomas at various stages of maturation and with different structural characteristics. Such enamel was sometimes associated with layers of ameloblastic cells that appeared to have the characteristics of functionally active cells (Figs. 3F, 4C). Furthermore, areas of epithelial columnar ameloblast-like cells with centrally located nuclei, resembling secretory ameloblasts, were found in a number of compound and complex odontomas. These cells showed strong membranous staining for podoplanin (Fig. 4C). Ghost cells with granular cytoplasm were partially positive for podoplanin, and were generally associated with epithelial components or in contact with tubular dentin and calcified materials (Figs. 4D, E).
Occasionally, adherent epithelium to ghost cells showed strong membranous staining for podoplanin (Fig 4D).

Osteocytes in bone-like tissue were positive for podoplanin in all specimens. In cases of impacted teeth, membranous podoplanin positivity was found in superficial odontoblast layers. Tomes' fibers within the predentin and pulp cells associated with odontoblasts were podoplanin-positive (Fig. 4F).

\section{Discussion Clinicopathological findings}

The morphology of odontomas provides an alternative model for observing the formation of dental tissues, since different stages of maturation are present simultaneously.

The term odontoma was originally used as a general term for any tumor of dental tissue origin (3). Through time, however, it has come to be employed in a much more restricted sense. It is now included in the current WHO (2) classification of head and neck tumors, as a group of lesions affecting the odontogenic epithelium with odontogenic ectomesenchyme, with or without hard tissue formation. Odontomas include two types: complex and compound. In the present study, to gain information on the possible pathogenesis of these malformations, the two types were separated for comparative clinicophatological examination.

Complex odontoma is defined in the 2005 WHO classification as a tumor-like malformation (hamartoma) in which enamel and dentin, and sometimes cementum, are present (2). Odontomas are common odontogenic tumors that affect primarily children, adolescents and young adults. In their extensive review of the literature and personal files, Philipsen et al. (3) found a median mean age at occurrence of 19.9 years. In the present series, patients with complex odontomas showed a considerably higher mean age (30.5 years) than in series reported previously. However, complex odontoma can be considered a lesion affecting young patients, because the majority of the cases occurred before the age of 30 , with a peak in the second decade of life. The WHO and some previous studies have reported no gender predilection for odontomas $(2,15)$. However, in a literature review and analysis of personal files, Hidalgo-Sánchez et al. (16) found a slight female gender predilection, similarly to our findings. The most common location was the posterior mandible, followed by the anterior maxilla, being similar to previous reports of complex odontoma (1-3).

Compound odontoma is defined as a tumor-like malformation (hamartoma) with varied numbers of toothlike elements (2). It has been reported to be the most common of all odontogenic neoplasms and tumor-like 
lesions; however, keratocystic odontogenic tumor is the most common odontogenic tumor diagnosed in our laboratory. Compound odontoma is diagnosed primarily in children and adolescents. The majority of the cases in the present study appeared before the age of 20 years, with a peak in the second decade of life, similarly to previous findings $(3,17)$. Philpsen et al. found that compound odontomas were slightly more common in males than in females, similarly to the present data demonstrating a slight male gender predilection (1.4:1). The anterior maxilla appears to be the site most frequently affected (2,3,15-17), as reflected in the findings of the present study.

Some authors have considered that differences in patient age and site distribution between the two types of odontoma support the hypothesis that the two lesions have different pathogenesis $(1,3)$. It has been suggested that complex odontomas represent the terminal stage of a hamartomatous lesion, whereas compound odontomas are malformations that result from multiple tooth formation due to locally conditioned hyperactivity of the dental lamina $(1,3)$.

The reported incidence of ghost cells in odontomas has ranged from $11 \%$ to $66.7 \%$ (17-20). In the present series, these were observed in $41.4 \%$ of the complex and $66.7 \%$ of the compound odontomas, which was a relatively high incidence. Although the reported incidence of ghost cells in complex odontomas is higher than in compound odontomas (17-19), the present study revealed a higher incidence of these cells in compound odontomas than in complex odontomas. The process of calcification in ghost cells has been described in various studies (20-23). In general, dysplastic calcified tissues in less differentiated odontomas present different degrees of calcification (22).

A review of the literature indicates that two different kinds of dysplastic calcified tissue have been consistently noted. One type of calcification has a concentric arrangement and is similar to the type formed by Liesegang's rings, being in close contact with epithelial cells of the intermediate layers and of the stellate reticulum of the enamel organ (20-23). The second type of calcification does not form concentric layers; the material is uniform and appears to be derived from amyloid-like material produced by ghost cells (20-23). There are some reported cases in which ghost cells showed intracytoplasmic calcification or had been substituted completely by dysplastic calcified materials (20-23). We were able to identify the same patterns of calcification in the cases we examined. Taken together, the data suggest that in odontomas two different types of dysplastic calcifying cells are present, and that these cells produce two different tissues, unlike normal dental mineralized tissues (22).

\section{Immunohistochemical findings}

Immunoreactivity for podoplanin was found in $55.2 \%$ (16 cases) of the complex odontomas and in 71.9\% (41 cases) of the compound odontomas. The degree of morphodifferentation varied from lesion to lesion, but positive localization of podoplanin was consistent with the cell type. Expression was evident in differentiating and mature odontoblasts, secretory polarized cells, ameloblasts, Tomes' fibers, and pulp cells associated with odontoblasts, as well as in stellate reticulum-like cells, although weakly. In developing, and in less differentiated, odontomas, podoplanin expression was detected in enamel epithelia of the cervical loop at cell-cell contacts, and in odontoblasts along with their dentin tubular extensions.

However, some cases, including odontoblasts and pulp cells mimicking those of intact permanent teeth, did not express podoplanin. In this respect, podoplanin expression was less evident in complex odontomas than in compound odontomas, probably due to the extensive calcified tissues and few epithelial components. In addition, it is possible that the decalcification procedure employed may have destroyed the molecular structure of the antigen.

Our findings are in line with recent observations by Sawa et al. (5), who described podoplanin expression in mouse tooth germ and apical bud, and those by Zustin et al. (12) in human tooth germ. The present data corroborate earlier evidence that podoplanin is expressed in the enamel epithelia of the cervical loop, and in differentiating odontoblasts, being sustained in the latter, indicating their marked ability to express podoplanin. In addition, podoplanin expression is lacking in ameloblasts of completely developed human and murine teeth $(5,12)$, and also in mature ameloblasts in odontoma tissues. Furthermore, the osteocytes in bone-like structures within odontomas were also positive for podoplanin, similar to previous findings in osteoid tissues $(6,23)$.

Podoplanin associated with odontogenic ectomesenchyme is not expressed throughout life, but rather during specific periods such as odontogenesis (5). The presence of podoplanin in odontoblasts and secretory ameloblasts of odontomas would therefore suggest that they correspond to the cap and/or the early bell stages of normal tooth development. The pattern of staining found in odontomas is similar to the transient co-expression of podoplanin that also occurs in the developing tooth.

The loss of podoplanin in mature ameloblasts might be related to the maturation of enamel. When ameloblasts begin secretion, they exhibit cyclical variations in shape, with a ruffled end and a smooth end; the cells move away from the dentin, leaving behind layers of enamel. Such matrix deposition and mineralization continue peripherally 
to the tips of the cusps, and then laterally on the sides of the crown (24). The strongest expression of podoplanin in ameloblasts occurs during this phase $(5,12)$. Probably podoplanin, with its ability to remodel the actin cytoskeleton and form filipodia, is involved in the movement of ameloblasts away from odontoblasts, and vice versa. Once enamel deposition has been completed and the ameloblasts no longer move, they lose their podoplanin expression. Odontoblasts, however, continue to form dentin and secondary dentin after the completion of tooth development, and need motility; hence they continue to express podoplanin.

Analysis of the differentiation of normal and tumorrelated odontogenic cells has provided new insight into the processes of odontogenesis. In the odontomas analyzed in the present study, the same secretory ameloblast-like cells showed strong membranous staining for podoplanin, and this expression was lost in mature ameloblasts, similar to features observed during normal tooth development $(5,12)$. As ameloblasts complete the matrix deposition phase, they change in both appearance and function, and lose their expression of podoplanin not only in the tooth germ, but also in odontomas.

The present study revealed a high percentage of ghost cells in complex and compound odontomas, but only a few ghost cells with granular cytoplasm were positive for podoplanin and, sometimes, adherent epithelium to ghost cells showed strong staining for podoplanin. It is possible that epithelial cells expressing podoplanin enlarge and undergo metaplastic transformation relatively quickly, and that the protein is trapped within the metaplastic cells, rather than being actively involved in ghost cell formation.

In addition, the expression of podoplanin in cells with a mixed epithelio-mesenchymal phenotype in odontomas suggests that some transient and specific events probably occur during normal tooth development, but at an undetectable level. It is possible that podoplanin mediates transmembrane signaling events by interacting with other surface glycoproteins, similar to the clustering and activation of integrins. However, there is no apparent homology between podoplanin and any other protein domains (25). Therefore, podoplanin may interact in pathways involving yet unknown binding partners, including soluble ligands or cell surface molecules interacting with podoplanin's carbohydrate moiety or signaling effector molecules within the cell $(14,25)$.

The role of podoplanin in development and tumor progression remains unclear. Enhanced podoplanin expression induces the formation of filipodia-like plasma membrane extensions in a variety of cell types, and a conserved domain mediates its interactions with the membrane cytoskeleton linkers ezrin and moesin (25).

Wicki et al. (8) have found that epithelial growth factor, fibroblast growth factor- 2 , and tumor growth factor- $\beta$ can upregulate the expression of podoplanin in MCF7 cells; their data suggest that stroma-derived growth factors may induce the specific expression of podoplanin (25).

The distribution of podoplanin in odontomas suggests that its expression is upregulated by interactions between odontogenic epithelium and mesenchyme; it could also be related to epithelial-mesenchymal transition, in which epithelial cells lose their polarity and cohesiveness and acquire migratory features and the characteristics of fibroblasts. In addition, podoplanin could be involved in the differentiation of pulp cells to odontoblasts in odontomas.

\section{References}

1. Philipsen HP, Reichart PA (2004) Benign neoplasms and tumor-like lesions showing odontogenic epithelium with odontogenic ectomesenchyme, with or without dental hard tissue formation. In: Odontogenic tumors and allied lesions, Quintessence Publishing, London, 141-153.

2. Prætorius F, Piattelli A (2005) Odontogenic tumours. In: WHO classification of tumours. Pathology \& genetics. Head and neck tumours, Barnes L, Evenson JW, Reichart PA, Sindransky D eds, IARC Press, Lyon, 310-311.

3. Philipsen HP, Reichart PA, Praetorius F (1997) Mixed odontogenic tumours and odontomas. Considerations on interrelationship. Review of the literature and presentation of 134 new cases of odontomas. Oral Oncol 33, 86-99.

4. Wicki A, Christofori G (2007) The potential role of podoplanin in tumor invasion. Br J Cancer 96, 1-5.

5. Sawa Y, Iwasawa K, Ishikawa H (2008) Expression of podoplanin in the mouse tooth germ and apical bud cells. Acta Histochem Cytochem 41, 121-126.

6. Schacht V, Dadras SS, Johnson LA, Jackson DG, Hong YK, Detmar M (2005) Up-regulation of the lymphatic marker podoplanin, a mucin-type transmembrane glycoprotein, in human squamous cell carcinomas and germ cell tumors. Am J Pathol 166, 913-921.

7. Kawaguchi H, El-Naggar AK, Papadimitrakopoulou V, Ren H, Fan YH, Feng L, Lee JJ, Kim E, Hong WK, Lippman SM, Mao L (2008) Podoplanin: a novel marker for oral cancer risk in patients with oral premalignancy. J Clin Oncol 26, 354-360.

8. Wicki A, Lehembre F, Wick N, Hantusch B, Kerjaschki D, Christofori G (2006) Tumor invasion 
in the absence of epithelial-mesenchymal transition: podoplanin-mediated remodeling of the actin cytoskeleton. Cancer Cell 9, 261-272.

9. Oku Y, Tanaka A, González-Alva P, Sakashita H, Kusama K (2008) Podoplanin expression in human pleomorphic adenomas. In: Oral oncology, Vol 12, Varma AK, Qiu WL, Zhang CP, Zhang ZP eds. Ocean Papers \& Printers, Shanghai, 251-253.

10. Okamoto E, Kikuchi K, Miyazaki Y, GonzálezAlva P, Oku Y, Tanaka A, Yoshida N, Fujinami M, Ide F, Sakashita H, Kusama K (2010) Significance of podoplanin expression in keratocystic odontogenic tumor. J Oral Pathol Med 39, 110-114.

11. González-Alva P, Tanaka A, Oku Y, Miyazaki Y, Okamoto E, Fujinami M, Yoshida N, Kikuchi K, Ide F, Sakashita H, Kusama K (2010) Enhanced expression of podoplanin in ameloblastomas. J Oral Pathol Med 39, 103-109.

12. Zustin J, Scheuer HA, Friedrich RE (2010) Podoplanin expression in human tooth germ tissues and cystic odontogenic lesions: an immunohistochemical study. J Oral Pathol Med 39, 115-120.

13. Miyazaki Y, Okamoto E, González-Alva P, Hayashi J, Ishige T, Kikuchi K, Nemoto N, Shin K, Sakashita H, Ochiai K, Kusama K (2009) The significance of podoplanin expression in human inflamed gingiva. J Oral Sci 51, 283-287.

14. King RE, Altimi M, Shear M (1979) Basal cell extensions in human oral mucosa. J Oral Pathol 8, 140-146.

15. Mosqueda-Taylor A, Ledesma-Montes C, CaballeroSandoval S, Portilla-Robertson J, Ruíz-Godoy Rivera LM, Meneses-García A (1997) Odontogenic tumors in Mexico: a collaborative retrospective study of 349 cases. Oral Surg Oral Med Oral Pathol Oral Radiol Endod 84, 672-675.
16. Hidalgo-Sánchez O, Leco-Berrocal MI, MartínezGonzález JM (2008) Metaanalysis of the epidemiology and clinical manifestations of odontomas. Med Oral Patol Oral Cir Bucal 13, E730-734.

17. Tanaka A, Okamoto M, Yoshizawa D, Ito S, Alva PG, Ide F, Kusama K (2007) Presence of ghost cells and the Wnt signaling pathway in odontomas. J Oral Pathol Med 36, 400-404.

18. Sedano HO, Pindborg JJ (1975) Ghost cell epithelium in odontomas. J Oral Pathol 4, 27-30.

19. Regezi JA, Kerr DA, Courtney RM (1978) Odontogenic tumors: analysis of 706 cases. J Oral Surg 36, 771-778.

20. Katz RW (1989) An analysis of compound and complex odontomas. ASDC J Dent Child 56, 445449.

21. Kerebel B, Kerebel LM (1985) Ghost cells in complex odontoma: a light microscopic and SEM study. Oral Surg Oral Med Oral Pathol 59, 371-378.

22. Piatelli A, Trisi P (1992) Morphodifferentiation and histodifferentiation of the dental hard tissues in compound odontoma: a study of undermineralized material. J Oral Pathol Med 21, 340-342.

23. Piatelli A, Trisi P (1991) Ghost cells in compound odontoma: a study of undemineralized material. Bull Group Int Rech Sci Stomatol Odontol 34, 145149.

24. Nanci A (2008) Ten Cate's oral histology. Development, structure, and function. 7th ed, Mosby Elsevier, St Louis, 79-107.

25. Martín-Villar E, Megías D, Castel S, Yurrita MM, Vilaró S, Quintanilla M (2006) Podoplanin binds ERM proteins to activate RhoA and promote epithelial-mesenchymal transition. J Cell Sci 119, 4541-4553. 